\title{
Induced pluripotent stem cell lines from Huntington's disease mice undergo neuronal differentiation while showing alterations in the lysosomal pathway
}

\author{
Valentina Castiglioni, Marco Onorati, Christelle Rochon ${ }^{1}$, Elena Cattaneo * \\ Department of Pharmacological Sciences and Centre for Stem Cell Research, Università degli Studi di Milano, Via Balzaretti 9-20133 Milano, Italy
}

\section{A R T I C L E I N F O}

Article history:

Received 21 July 2011

Revised 13 December 2011

Accepted 17 December 2011

Available online 28 December 2011

\section{Keywords:}

Induced pluripotent stem cells

Huntington's disease

Neuronal differentiation

$\mathrm{R} 6 / 2$

\begin{abstract}
A B S T R A C T
Huntington's disease (HD) is an autosomal dominant neurodegenerative disorder caused by an excessive expansion of a CAG trinucleotide repeat in the gene encoding the protein huntingtin, resulting in an elongated stretch of glutamines near the N-terminus of the protein. Here we report the derivation of a collection of 11 induced pluripotent stem (iPS) cell lines generated through somatic reprogramming of fibroblasts obtained from the R6/2 transgenic HD mouse line. We show that CAG expansion has no effect on reprogramming efficiency, cell proliferation rate, brain-derived neurotrophic factor level, or neurogenic potential. However, genes involved in the cholesterol biosynthesis pathway, which is altered in HD, are also affected in HD-iPS cell lines. Furthermore, we found a lysosomal gene upregulation and an increase in lysosome number in HD-iPS cell lines. These observations suggest that iPS cells from HD mice replicate some but not all of the molecular phenotypes typically observed in the disease; additionally, they do not manifest increased cell death propensity either under self-renewal or differentiated conditions. More studies will be necessary to transform a revolutionary technology into a powerful platform for drug screening approaches.
\end{abstract}

(C) 2011 Elsevier Inc. All rights reserved.

\section{Introduction}

Huntington's disease (HD) is an untreatable, progressive genetic neurodegenerative disorder caused by an unstable expansion of the CAG repeat within the coding region of the IT-15 gene. This gene encodes for the huntingtin (HTT) protein and the mutation results in an elongated stretch of glutamines in the N-terminus of the protein (HD Collaborative Research Group, 1993). This HTT mutation is responsible for massive brain neurodegeneration characterized by the prevalent loss of efferent medium spiny neurons in the striatum, the main input station of the basal ganglia circuit, but progressively involves cortical neuronal structures as well (Reiner et al., 1988; Rosas et al., 2003, 2005, 2008).

HD is characterized by chorea, cognitive abnormalities, and psychiatric disturbances that manifest in mid-adulthood and progress inexorably toward death. The CAG expansion in HTT is the triggering

Abbreviations: iPS, induced pluripotent stem cells; HD, Huntington's disease; wt wild type; HTT, huntingtin; BDNF, brain-derived neurotrophic factor; PH3, phosphohistone H3; PI, propidium iodide; GFAP, glial fibrillary acid protein; MAP2ab, microtubule-associated protein $2 \mathrm{ab}$; GAP-43, growth associated protein 43; Hmgcr, 3-hydroxy3-methylglutaryl-Coenzyme A reductase; 7dhcr, 7-dehydrocholesterol reductase; Cyp51, cytochrome P450 Lanosterol 14 alpha-demethylase; TFEB, Transcription Factor EB; Ctsf, cathepsin F; Tpp1, tripeptidyl peptidase; Lamp1, lysosomal-associated membrane protein; TTF, tail-tip fibroblasts; MEF, mouse embryonic fibroblasts; ES, embryonic stem.

* Corresponding author. Fax: + 390250325843.

E-mail address: elena.cattaneo@unimi.it (E. Cattaneo).

${ }^{1}$ Current address: ECTYCELL SASU, 4 rue Pierre Fontaine, 91000 Evry, France.

Available online on ScienceDirect (www.sciencedirect.com). event that endows the protein with new toxic functions that are deleterious to striatal and cortical neurons. At the same time, the specificity of the neuronal loss may be due to the protein context in which the CAG is expanded (Cattaneo, 2001); the mutation also affects the ability of normal HTT to exert beneficial activities for the neurons that degenerate in HD (Zuccato et al., 2010).

While our understanding of HD pathophysiology is advancing rapidly, our knowledge is still incomplete. Since HD is caused by a single mutation, introduction of the mutant gene into non-human primates, mice, flies, fishes, and worms has generated disease models that have been extremely valuable in the identification of pathways affected by the mutation and as validation tools for ex vivo-identified targets (Zuccato et al., 2010). In parallel, since the year 2000 we and others have reported the generation of a large collection of brain-derived cell lines from rodents, including the first clones of immortalized ST14A cells carrying the full length or truncated normal or mutant HTT gene (Rigamonti et al., 2000), their inducible variants (Sipione et al., 2002), knock-in cell lines (Trettel et al., 2000), and, more recently, rodent- and human-derived neural stem cell lines carrying the mutant HTT gene (unpublished results).

Collectively, these and other cell lines have been instrumental in providing the first indications of a loss-of-HTT-function mechanism operating in HD, and were used to deliver the first evidence of losses in BDNF mRNA and protein levels and reduced transcription of other NRSE-RE1-controlled neuronal genes (Rigamonti et al., 2007; Zuccato et al., 2003, 2007). In addition, gene expression profiling of ST14A cell line stably expressing an inducible mutant HTT construct revealed a defect in the cholesterol biosynthesis pathway that was confirmed 
in multiple mouse models; that study is currently the focus of both investigations and debate (Valenza and Cattaneo, 2011; Valenza et al., 2010).

Importantly, somatic reprogramming technology (Blelloch et al., 2007; Takahashi and Yamanaka, 2006; Takahashi et al., 2007) has yielded iPS cells with the potential to bridge clinical knowledge in patients with the molecular and biological expertise in genetically precise human cell lines that, upon differentiation, should more closely resemble the neurons affected by neurodegenerative disease. The generation of patient-specific cell models offers a number of advantages for neurological diseases research (Abeliovich and Doege, 2009; Brennand et al., 2011; Dimos et al., 2008; Ebert et al., 2009; Park et al., 2008; Soldner et al., 2009), although this technology may be limited by the difficulty of recapitulating disease progression in a dish.

Here we report the generation of a collection of 40 mouse iPS cell clones derived from two fibroblast lines obtained from R6/2 mice (HD-iPS cell lines) and 20 iPS cell lines from one wild type fibroblast line (the wt-iPS cell line). R6/2 mice were generated by insertion of a 1.9-kb DNA fragment containing the human HTT promoter and exon 1 of the human HTT gene bearing 144 CAG repeats (Mangiarini et al., 1996). These mice exhibit severe behavioral and anatomical symptoms, including widespread nuclear inclusions of mutant HTT in neurons (Davies et al., 1997; Morton, 2000) and typical gene expression dysregulation (Apostol et al., 2008; Conforti et al., 2008; LuthiCarter et al., 2000, 2002; Sadri-Vakili et al., 2007; Sipione et al., 2002; Tarditi et al., 2006; Valenza et al., 2007; Zuccato et al., 2001, 2005, 2010). We analyzed these wt- and HD-iPS cells and found that while somatic reprogramming is not affected by the HD mutation, typical HD cellular features are present in HD-iPS cells but not in the wt-iPS lines, mainly involving transcriptional dysregulation in cholesterol biosynthetic genes and lysosomal pathway activation.

\section{Materials and methods}

iPS cell generation

The day before transfection, PLAT-E cells (Morita et al., 2000) were seeded at a density of $4 \times 10^{6}$ cells $/ 100-\mathrm{mm}$ dish. The next day, pMXsbased retroviral vectors (Klf4, Sox2, c-Myc, and Oct4, Addgene plasmid $13370,13367,13375,13366$, respectively) were introduced into PLAT-E cells using Lipofectamine 2000 (Invitrogen) according to the manufacturer's recommendations. Six hours after transfection, the medium was replaced with PLAT-E medium without selection.

TTFs were seeded at $1.2 \times 10^{5}$ cells/6-well plate. After $24 \mathrm{~h}$, viruscontaining supernatants derived from PLAT-E cultures were filtered through a $0.45 \mu \mathrm{m}$ cellulose acetate filter (Millipore). Equal amounts of viral supernatants were mixed and supplemented with $4 \mu \mathrm{g} / \mathrm{ml}$ of polybrene (Millipore). TTFs were incubated in the virus/polybrenecontaining supernatants for $48 \mathrm{~h}$, then restored to fibroblast medium for $24 \mathrm{~h}$. Three days after infection the fibroblast medium was replaced with mouse ES cell medium; 48 h later, infected TTFs were transferred on mitomycin C-treated CD1 feeder layer. The medium was replaced every two days. The described iPS cell lines can be requested from the cell repository at Biorep S.r.l. (http://www. biorep.it/eng/).

\section{Cell culture}

PLAT-E cells were used to produce retroviruses and were maintained in DMEM containing 10\% FBS (Euroclone), $1 \mu \mathrm{g} / \mathrm{ml}$ puromycin (Sigma), and $10 \mu \mathrm{g} / \mathrm{ml}$ blasticidin S (Sigma). iPS and mES R1 cells were cultured in GMEM (Sigma) supplemented with 10\% heatinactivated FBS, $1 \times$ Penicillin/Streptomycin (Euroclone, Italy), $2 \mathrm{mM}$ L-Glutamine (Euroclone, Italy), $1 \times$ non-essential amino acids (Gibco), $1 \mathrm{mM}$ sodium pyruvate (Gibco), $0.1 \mathrm{mM} \beta$-mercapto-ethanol (Gibco), and $1000 \mathrm{U} / \mathrm{ml}$ murine leukemia inhibitory factor (LIF-ESGRO, Millipore). Mitomycin C-inactivated CD1 mouse embryonic fibroblasts were used as a feeder layer for the expansion of iPS cells.

To establish TTFs, the tails from adult mice were peeled, minced into $1 \mathrm{~cm}$ pieces, and placed onto gelatin-coated culture dishes in fibroblast medium (DMEM, 10\% FBS, 1× Penicillin/Streptomycin (Euroclone, Italy)), $2 \mathrm{mM}$ L-Glutamine (Euroclone, Italy) for five days. Cells that migrated out of the graft pieces were transferred into new plates and maintained in fibroblast medium. We infected TTFs at passage 3 for iPS cell induction.

\section{Alkaline phospatase staining}

Three weeks after infection wt- and HD-iPS cells were stained for alkaline phosphatase using SIGMA FAST ${ }^{T M}$ BCIP/NBT (Sigma) according to the manufacturer's instructions.

\section{Embryoid bodies}

iPS and ES cells were detached using a $0.025 \%$ trypsin (Gibco) solution at $37^{\circ} \mathrm{C}$ and plated onto bacterial culture dishes at a density of $1 \times 10^{6}$ cells/plate in supplemented GMEM without leukemia inhibitory factor. After five days, cell aggregates were plated onto gelatincoated culture dishes for another six days and then fixed for analysis.

\section{Lysotracker $\circledR^{\circledR}$ staining}

Proliferating wt- and HD-iPs cells were incubated with Lysotracker ${ }^{\circledR}$ dye for $30 \mathrm{~min}$ at $37^{\circ} \mathrm{C}$ according to the manufacturer's instructions. Lysotracker ${ }^{\circledR}$-positive dots were counted using Image software (Rasband, W.S., ImageJ, U. S. National Institutes of Health, Bethesda). Three fields per clone were counted. ${ }^{*} p<0.05,{ }^{* *} p<0.01$, ${ }^{* * *} p<0.001$, as calculated by one-way ANOVA followed by Dunnett post-hoc test.

\section{Monolayer neural differentiation of mouse iPS and ES cells}

Cells were detached using a $0.025 \%$ trypsin solution at $37{ }^{\circ} \mathrm{C}$ and plated onto gelatin-coated 6-well plates or T25 flasks in N2B27 serum-free medium. N2B27 consists of 1:1 DMEM/F12 (Gibco) and Neurobasal (Gibco) media supplemented with 0.5\% N2 (Gibco), 1\% B27 (Gibco), and 0.2\% $\beta$-mercapto-ethanol. The medium was changed every two days. At day 7-10, cells were replated onto laminin (Invitrogen)-coated 6 -well plates at a density of $6 \times 10^{5}$ cells/well. Monolayer cultures were kept under differentiation until day 21 .

\section{Cell genotyping}

DNA was extracted from cells using NucleoSpin kit (MacherayNagel) according to the manufacturer's instructions. The CAG expansion was amplified by PCR using primer 31329 (5'-ATGAAGGCCTTCGAGTCCCTCAAGTCCTTC-3') and primer 33934 (5'-GGCGGCTGAGGAAGCTGAGGA-3' in AM buffer (67 mM Tris-HCl [pH 8.8], $16.6 \mathrm{mM}$ $\mathrm{NH}_{4} \mathrm{SO}_{4}, 2 \mathrm{mM} \mathrm{MgCl}, 0.17 \mathrm{mg} / \mathrm{ml}$ bovine serum albumin, $10 \mathrm{mM}$ $\beta$-mercapto-ethanol), 10\% dimethylsulfoxide, $200 \mu \mathrm{M}$ dNTPs, $8 \mathrm{ng} / \mu \mathrm{l}$ primers, and $0.5 \mathrm{U} / \mu \mathrm{l}$ Taq polymerase (FINNZYMES). Cycling conditions were $95^{\circ} \mathrm{C}$ for $3 \mathrm{~min}, 45$ cycles of $30 \mathrm{~s}$ at $95^{\circ} \mathrm{C}, 30 \mathrm{~s}$ at $65^{\circ} \mathrm{C}, 90 \mathrm{~s}$ at $72{ }^{\circ} \mathrm{C}$, and 7 min at $72{ }^{\circ} \mathrm{C}$.

\section{RNA isolation}

Total RNA was extracted from iPS cells with Trizol reagent (Invitrogen) according to the manufacturer's recommendations. Before reverse transcription, RNA was treated with DNase I (Qiagen) and purified using the RNeasy kit (Qiagen). After DNase treatment, $1 \mu \mathrm{g}$ of total RNA was reverse transcribed to single-stranded cDNA using 
SuperScript III reverse transcriptase and random primers in a total volume of $20 \mu \mathrm{l}$ according to the manufacturer's instruction (Invitrogen).

\section{$R T-P C R$}

RT-PCR was performed in a total volume of $25 \mu \mathrm{l}$ containing $25 \mathrm{ng}$ cDNA, $50 \mathrm{mM} \mathrm{KCl}, 1.5 \mathrm{mM} \mathrm{MgCl} 2,0.2 \mathrm{mM}$ dNTPs, $0.4 \mu \mathrm{M}$ of each primer, and $1 \mathrm{U}$ Taq polymerase (Invitrogen). RT-PCR was performed for 30 cycles for all markers as follows: denaturing for $30 \mathrm{~s}$ at $94^{\circ} \mathrm{C}$, annealing for $30 \mathrm{~s}$ (Table S1), and extension for $45 \mathrm{~s}$ at $72^{\circ} \mathrm{C}$. PCR products were resolved on a $2 \%$ agarose gel. Primer sequences, annealing temperatures, and product sizes are listed in Table S1.

\section{Quantitative real-time $R T-P C R$}

Two independent reverse transcriptase reactions were set up for every RNA sample. Quantitative real-time RT-PCR (RT-qPCR) was performed in triplicate for the analyzed genes using the iCycler Thermal Cycler with the Multicolor RT-PCR Detection System (Biorad). All reactions were performed in a total volume of $25 \mu \mathrm{l}$ containing $50 \mathrm{ng}$ cDNA, $50 \mathrm{mM} \mathrm{KCl}, 20 \mathrm{mM}$ Tris- $\mathrm{HCl}$ [pH 8.4], $0.2 \mathrm{mM}$ dNTPs, $25 \mathrm{U} / \mathrm{ml}$ iTaq DNA polymerase, $3 \mathrm{mM} \mathrm{MgCl}_{2}$, SYBR Green I (Biorad), $10 \mathrm{nM}$ fluorescein, stabilizer $\mathrm{iQ}^{\mathrm{TM}}$ SYBR Green Supermix (Bio-Rad), and $0.3 \mathrm{mM}$ forward and reverse primers. Amplification cycles consisted of a first denaturating cycle at $95^{\circ} \mathrm{C}$ for 3 min, followed by 45 cycles of $30 \mathrm{~s}$ at $95^{\circ} \mathrm{C}$ and $30 \mathrm{~s}$ at $60{ }^{\circ} \mathrm{C}$. The amounts of target gene mRNA were normalized to $\beta$-actin levels. Primer sequences, annealing temperatures, and product sizes are listed in Table S1. Statistical analysis was performed by means of one-way ANOVA and Dunnett post-hoc test.

\section{Immunofluorescence}

Cells were fixed in $4 \%$ paraformaldehyde for $15 \mathrm{~min}$. For labeling intracellular antigens, cells were permeabilized for 15 min with $0.5 \%$ Triton X-100 (Euroclone). After washing in PBS, cells were blocked with 5\% FBS in PBS for $1 \mathrm{~h}$ and incubated overnight at $4{ }^{\circ} \mathrm{C}$ in $3 \% \mathrm{FBS}$ in PBS with the following primary antibodies: anti-Nanog, 1:500, (Novus Biological); anti-OCT4, 1:100, (Santa Cruz); anti-E-cadherin,

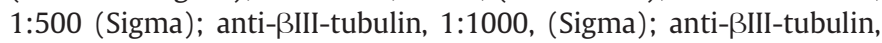
1:1000, (Covance); anti- $\alpha$ smooth muscle actin, 1:800, (Sigma); anti-GATA4, 1:200, (Santa Cruz); anti-nestin, 1:200, (Millipore); anti-N-cadherin, 1:500, (BD Bioscience); anti-MAP2ab, 1:350, (BD Bioscience); anti-GAP43, 1:500, (Chemicon); anti-HTT protein, clone mEM48, 1:50, (Chemicon); anti-Ki-67, 1:500, (Abcam); anti-PH3, 1:100, (Cell Signaling). After two washes in PBS, appropriate secondary antibodies conjugated to Alexa fluorophores 488 or 568 (Molecular Probes, Invitrogen) were diluted 1:500 in blocking solution and mixed with Hoechst 33258 ( $5 \mu \mathrm{g} / \mathrm{ml}$; Molecular Probes, Invitrogen) to counterstain the nuclei. Images were acquired with a Leica DMI 6000B microscope $(20 \times$ objective) with LAS-AF imaging software and then processed using Adobe Photoshop.

\section{Western blotting}

Cells were homogenized in RIPA buffer (tris $\mathrm{HCl}$ pH8 $50 \mathrm{mM}, \mathrm{NaCl}$ $150 \mathrm{mM}$, SDS $0.1 \%$, NP40 1\%, Sodium deoxycholate 0.5\%) with PMSF $1 \mathrm{mM}$, DTT $0.5 \mathrm{mM}$ and protease inhibitor cocktail (Sigma). Protein was quantified using the BCA kit. $50 \mu \mathrm{g}$ of protein was loaded per track onto a 7.5\% SDS-PAGE gels. Protein were transferred onto a nitrocellulose membranes that were blocked in TBS-T with $5 \%$ non fat dry milk and immunoprobed with 3B5H10 (Sigma, 1:1000) and MAb2167 (Millipore, $1: 3000$ ) at $4{ }^{\circ} \mathrm{C}$ overnight. Washes were in TBS-T. Secondary antibody probing and detection was by use of the ECL kit (Pierce).

\section{MTT analysis}

Cells were plated in triplicate into 24 -well plates at a density of $2 \times 10^{4}$ cells/well in supplemented GMEM as indicated above. All cells were then incubated at $37^{\circ} \mathrm{C}$ for $6,12,24,36,48,60$ and $72 \mathrm{~h}$. At each time point the cells were exposed to Thiazolyl Blue Tetrazolium Bromide (Sigma), and formazan release was quantified at $560 \mathrm{~nm}$ using a plate reader (Synergy HT, BIOTEK).

\section{Flow cytometry}

Cells were propagated in an undifferentiated state on gelatincoated dishes. Cells were detached using a 0.025\% trypsin solution at $37{ }^{\circ} \mathrm{C}$ and centrifuged $3 \mathrm{~min}$ at $1200 \mathrm{rpm}$. Cells were fixed in $0.875 \mathrm{ml}$ cold PBS and $0.125 \mathrm{ml}$ cold $2 \%$ paraformaldehyde for 30-60 min at $4{ }^{\circ} \mathrm{C}$. Cells were permeabilized for $15 \mathrm{~min}$ with a $0.2 \%$ Tween 20 solution at $37^{\circ} \mathrm{C}$. Before incubation with primary antibodies, the pellet was resuspended in $50 \mu \mathrm{l}$ FBS and incubated for $1 \mathrm{~min}$. A $50 \mu \mathrm{l}$ solution containing primary antibodies (1:500 antiNanog, 1:200 anti-OCT4, 1:500 anti-Ki-67, and 1:100 anti-PH3) was added to the FBS-containing cell solution and incubated for $2 \mathrm{~h}$ at room temperature. Before incubation with secondary antibodies, the pellet was resuspended in $50 \mu \mathrm{l}$ FBS and incubated for 1 min. Fifty microliters of secondary antibodies (1:1000 AlexaFluor 488 and AlexaFluor 647) were added to the FBS solution and incubated for $30 \mathrm{~min}$ at room temperature. Cells incubated with primary isotypic antibody were used as a control. Flow cytometry was carried out with a FACS Canto II (BD Bioscience) and analyzed with BD FACSDiva v6.1.3 software.

\section{Propidium iodide analysis}

Cells were propagated in an undifferentiated state on gelatincoated dishes. Cells were detached using a $0.025 \%$ trypsin solution at $37^{\circ} \mathrm{C}$, pelleted, and then resuspended in $1 \mathrm{ml} 0.1 \%$ sodium citrate containing 0.1\% Nonidet P40, $6.2 \mu \mathrm{g} / \mathrm{ml}$ RNase, and $50 \mu \mathrm{g} / \mathrm{ml}$ PI (Invitrogen) and incubated in the dark for $4-12 \mathrm{~h}$. Flow cytometry was carried out with a FACS Canto II and analyzed with BD FACSDiva v6.1.3.

\section{Results}

\section{Generation and pluripotency evaluation of mouse HD-iPS cells}

TTFs were obtained from 10-week-old R6/2 mice and transduced with four reprogramming factors (Klf4, Sox2, c-Myc, and Oct4) in a single retroviral infection. After three weeks under mouse ES cellsupporting conditions, compact mouse ES-like colonies emerged from a background of fibroblasts, as previously described (Blelloch et al., 2007; Nakagawa et al., 2008; Takahashi and Yamanaka, 2006). HD-iPS cells were generated from two HD fibroblasts cell lines, HD2 and HD4, obtained from two different mice. In parallel, somatic reprogramming was carried on wt fibroblasts obtained from a single mouse. No differences in reprogramming efficiency or in clone morphology were observed between the two genotypes (Fig. 1A); in both cases, $\sim 1000$ colonies positive for alkaline phosphatase activity emerged after 20 days from $5 \times 10^{4}$ fibroblasts transduced with the four factors. A total of 60 iPS cell lines were subsequently isolated and grown, and among these11 HD-iPS (six lines derived from HD2 fibroblasts and five from HD4 fibroblasts) and $7 \mathrm{wt}$-iPS cell clones were randomly selected and further characterized for expression of pluripotency markers. Immunofluorescence revealed that the wtand HD-iPS cell clones were positive for nanog, OCT4, and Ecadherin protein expression (Fig. 1A). This antigenic profile was retained among passages ( 25 passages tested so far) and after adaptation to gelatin-coated dishes (data not shown). 
Next, we analyzed the mRNA expression of a panel of typical pluripotency genes via RT-PCR. All of the tested wt- and HD-iPS cell lines expressed Nanog, Fgf-4, E-cadherin, Cripto, and Rex1, as expected for fully reprogrammed iPS cells (Fig. 1B). Retroviral transgenes were largely silenced in all of the analyzed clones, with the exception of clones HD2-13 and HD2-16, which continued to exhibit transgene expression (Fig. 1B). The endogenous loci of the four reprogramming

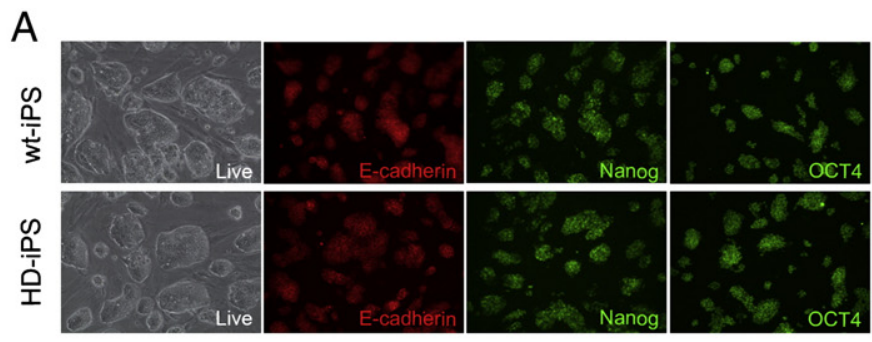

B
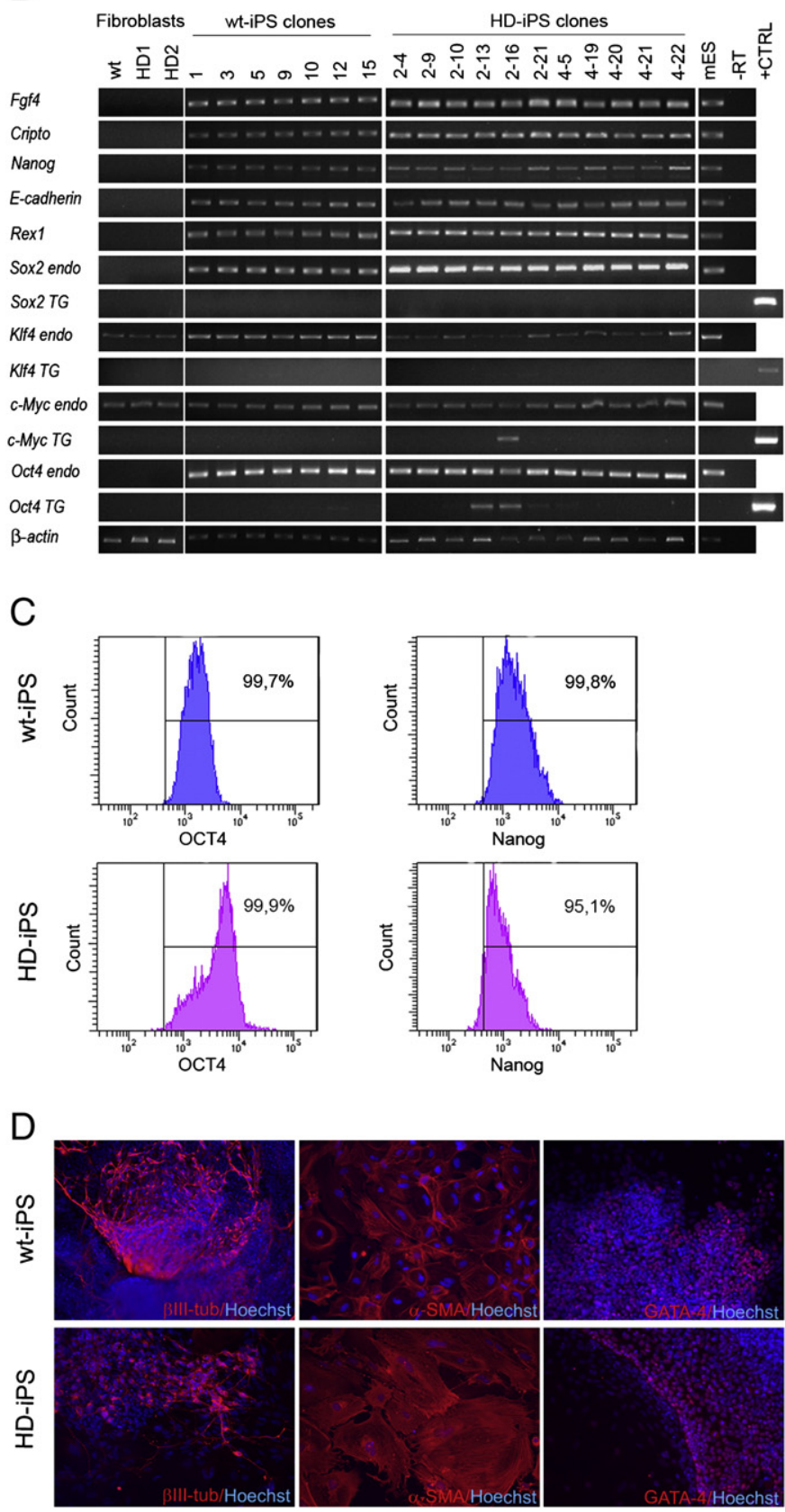

factors Klf4, Sox2, c-Myc, and Oct4 were reactivated in all clones analyzed (Fig. 1B). Out of our total set of 18 selected miPS cell clones, we performed flow cytometry on six wt- and six HD-iPS gelatinadapted iPS cell lines. We confirmed that $>95 \%$ of the cells were positive for nanog and OCT4 (Fig. 1C) protein expression, with no differences among the HD- and wt-iPS cell clones (Fig. 1C, Table S2).

In order to assay pluripotency, all 7 wt- and 11 HD-iPS cell lines were differentiated in vitro into embryoid bodies for 11 days (Fig. 1D). Embryoid bodies of similar size were obtained regardless of CAG expansion. In addition, immunofluorescence demonstrated that all wt- and HD-iPS cell lines were able to generate progeny of the three germ layers, as shown by the presence of cell derivatives positive for $\alpha$-smooth muscle actin (mesoderm), BIII-tubulin (ectoderm), and GATA-4 (endoderm; one representative example for each genotype appears in Fig. 1D).

Reprogramming did not grossly affect the length of the HD mutation in iPS cell clones, since an amplicon corresponding to 144 CAG repeats was retained in all lines (Suppl. Fig. 1) throughout passage (data not shown). RT-PCR and western blotting confirmed that HDiPS clones expressed normal and mutant HTT (Suppl. Figs. 1A and B).

\section{Mutant HTT does not impair proliferation and cell cycle progression}

We asked whether the CAG expansion affected cell division and cell cycle rate in self-renewing HD-iPS cells. We carried out the MTT assay to analyze the proliferation/survival rates of four HD-iPS cell lines in comparison to two wt-iPS cell lines and one mouse ES cell line. There were no substantial differences between the wt- and $\mathrm{HD}$-iPS cells, which exhibited the same proliferation rate as the mES cells (Fig. 2A). To further investigate the cell-cycle distribution in populations of wt- and HD-iPS cells, we performed flow cytometry for markers specific to the cell cycle phases. The percentage of PH3labeled cells in $M$ phase did not differ significantly ( $p>0.05$, oneway ANOVA) between one wt- and one HD-iPS cell line (Fig. 2B), an observation that was confirmed in another five lines for each of the two genotypes (Table S3). In both cases, $>96 \%$ of the cells were also positive for Ki-67, a marker of all active phases of the cell cycle (G1, $\mathrm{S}, \mathrm{G} 2$, and $\mathrm{M}$ ), demonstrating a coherent proliferative state (Fig. 2B). Finally, PI incorporation did not differ between wt- and HD-iPS cell lines in the various phases of the cell cycle (Fig. 2B, Table S3). Taken together, these observations demonstrate that the HD mutation has no effect on the cell cycle kinetics of self-renewing iPS cells.

\section{HD-iPS cells exhibit transcriptional dysregulation of some cholesterogenic and lysosomal genes}

R6/2 mice are characterized by wide expression dysregulation of genes encoding neuronal receptors such as adenosine A2a receptor, N-Methyl-D-aspartic acid (NMDA) receptors, dopamine receptors D1 and D2, metabotropic glutamate receptors mGluR1 and mGluR2 (Cha et al., 1998; Luthi-Carter et al., 2003), BDNF, and cholesterogenic genes (Leoni et al., 2011; Valenza et al., 2010; Zuccato et al., 2001, 2005). We evaluated the relative expression of Bdnf in wt- and HD-iPS

Fig. 1. Characterization of wt- and HD-iPS cells generated from 10-week-old wt and R6/2 mouse fibroblasts, respectively. A) Randomly selected iPS cell clones exhibit typical ES cell morphology and express the specific pluripotent markers Nanog, E-cadherin, and OCT4. B) RT-PCR of diagnostic pluripotency markers in six wt and 11 HD-iPS cell lines (six from HD2 and five from HD4 fibroblasts) showed a consistent gene expression profile. Retroviral transgenes (TG) were largely silenced in all analyzed clones (with two exceptions), while the endogenous loci (endo) of the four reprogramming factors were reactivated. Plasmid DNA was used as positive control $(+\mathrm{CTRL})$; $-\mathrm{RT}$ indicates the negative control for retrotranscription. C) Flow cytometry did not reveal differences in the percentage of OCT4- and Nanog-positive cells in the wt- and HD-iPS cell lines (C). D) Both wt- and HD-iPS cells lines were able to differentiate into derivatives of all three germ layers, as demonstrated by the expression of neuroectodermal ( $\beta$ III-tubulin), mesodermal $(\alpha-$ SMA), and endodermal (GATA-4) markers (D). 
cell clones, but we failed to detect any significant differences between the two genotypes under self-renewal conditions $(P>0.05$, one-way ANOVA and Dunnett post-hoc test; Fig. 3A). On the other hand, reprogramming led to an increase in the expression of cholesterogenic genes in the reprogrammed cells with respect to their parental fibroblasts (Suppl. Fig. 2). The finding of more prominent activity in the cholesterogenic pathway in pluripotent versus fibroblast cells (Suppl. Fig. 2) may reflect the known higher need for cholesterol synthesis in the early phases of embryonic development (Valenza and Cattaneo, 2011). In particular, compared to the fibroblasts from which they were derived, our iPS cell lines expressed higher mRNA levels for the genes encoding the rate-limiting enzymes of the cholesterol biosynthesis cascade: HMGCR, Cyp51 (an intermediate enzyme), and 7-DHCR (the last enzyme in the pathway; Valenza and Cattaneo, 2011) $(P<0.05$, one-way ANOVA and Dunnett post-hoc test; Suppl. Fig. 2). These measurements further confirm that reprogramming reschedules subtle biological activities in iPS cells.

We next pooled the seven wt-iPS and 11 HD-iPS cell clones into two groups according to the line of HD fibroblasts from which they were derived. RT-qPCR demonstrated a significant reduction in the mRNA levels of 7dhcr in all HD-iPS cell groups compared to the wtiPS cells $(P<0.05$, one-way ANOVA and Dunnett post-hoc test; Suppl. Fig. 2A; Fig. 3D), with very little intragroup variability. On the other hand, Cyp51 and Hmgcr mRNA levels in the HD-iPS cells were similar to the wt-miPS cells (Figs. 3B, C and Suppl. Figs. 3A and B).

Recently, it has been demonstrated that a relationship exists between lysosomal activity and HTT (Sardiello et al., 2009). The transcription factor EB (TFEB) was identified as a master regulator of a set of genes involved in autophagy and lysosomal biogenesis and function (Sardiello et al., 2009; Settembre et al., 2011), cellular programs that participate in macromolecule clearance (Ravikumar et al., 2002). Accordingly, TFEB overexpression in HD cells was found to increase the degradation of the mutant protein (Sardiello et al., 2009). We asked whether HD-iPS cells manifested altered mRNA levels of Tfeb and TFEB-regulated genes such as Tpp1, Ctsf, and Lamp1 versus control lines. RT-qPCR indicated that overall this pathway is more activated in HD-iPS cells, since a significant increase in the mRNA levels of Tpp1, Ctsf, and Tfeb were present in iPS cells carrying the HD mutation $(P<0.05$, one-way ANOVA and Dunnett post-hoc test; Figs. $3 \mathrm{E}-\mathrm{G}$ and Suppl. Figs. 2A and B). This transcriptional alteration was reflected by a significant increase in lysosome number in HD-iPS cells compared to wt-iPS cells, as demonstrated by staining with the lysosome-specific dye Lysotracker (Figs. 3I and J).

Taken together, these data demonstrate that HD-iPS cells recapitulate some HD phenotypes linked to transcriptional alteration of genes involved in cholesterol biosynthesis and lysosomal biogenesis. However, not all genes were coherently affected, since Cyp51, Hmgcr, and Lamp1 were not modulated. Surprisingly, Bdnf mRNA levels did not differ between the two genotypes, and although in most assays the HD2- and HD4-derived iPS cell lines behaved similarly, some differences, for example in Ctsf and Tpp1 mRNAs levels, were noted (Fig. 3F).

Neuronal derivatives of HD-iPS cells show altered lysosomal gene expression

To investigate whether the CAG expansion impacted in vitro neuronal differentiation potential and survival, we exposed wt- and HDiPS cells to a monolayer differentiation protocol able to efficiently neuralize pluripotent stem cells under serum-free conditions (Onorati et al., 2010; Ying et al., 2003). iPS cells and control mouse ES cells were plated at low density on gelatin-coated dishes; starting from day 8, neural progenitors appeared in the dish and were organized into neural rosettes. The latter were then dissociated and replated onto laminin-coated dishes for terminal differentiation until day 21 (Fig. 4). Individual wt- and HD-iPS cell lines exhibited a variable propensity to differentiate, with a delay in neural conversion of approximately two days compared to ES cells. This observation is in accordance with previous reports that various iPS cell lines have different neuronal differentiation properties and differentiation timing (Kim et al., 2010).

We selected two wt- and five HD-iPS cell lines with good neurogenic potential and further tested them for potential differences; however, three differentiation trials did not reveal major abnormalities in the ability of the HD-iPS cell clones to undergo neural conversion compared to the wt-iPS cells, as demonstrated by the parallel appearance of nestin-positive neural precursors and N-cadherinstained rosettes at day 10 in both genotypes (Fig. 4). Both wt- and HD-iPS cell lines differentiated toward BIII-tubulin-, MAP2ab-, and GFAP-positive cells in a comparable manner at days 10, 15, and 21 (Fig. 4 and Suppl. Fig. 4). We confirmed that the mutant HTT gene was efficiently transcribed during neural differentiation of the HDiPS cells, as demonstrated by RT-PCR of RNA samples from days 15 and 21 of the monolayer differentiation protocol (Suppl. Fig. 5). These observations suggest that the CAG expansion does not influence the neurogenic and gliogenic potential of cultured HD-iPS cells.

At day 0,15 and 21 of neuronal differentiation we also analyzed the expression profile of the same set of HD-related genes previously evaluated in self-renewal condition. We observed that the Bdnf mRNA levels significantly increased in both wt and HD clones during the neuronal differentiation with higher levels at day 21 respect to day $0(P<0.05$, one-way ANOVA and Bonferroni post-hoc test), but we did not observe differences among the wt and HD analyzed clones (Suppl. Fig. 6A). Furthermore, we did not observe differences among wt and HD iPS cell clones in the expression levels of CYP51, HMGCR and $7 D H C R$ (Suppl. Figs. 6B-D). Finally, we analyzed the expression of Tfeb and TFEB-regulated genes during neuronal differentiation finding a significant increase in the mRNA levels of Tfeb, Ctsf and Tpp1 in the HD iPS cell clones at day $21(P<0.05$, one-way ANOVA and Bonferroni post-hoc test; Figs. $5 \mathrm{~A}-\mathrm{C}$ ). On the other hand, we did not detect any difference in the mRNA levels of Lamp1 (Fig. 5D), as observed also in self-renewal condition.

\section{HTT inclusions appear during neuronal differentiation}

One of the characteristics of HD is the appearance of widespread nuclear and cytoplasmic inclusions of mutant HTT in neurons that increase in number, size, and distribution as the disease progresses (Davies et al., 1997; Morton, 2000). We tested whether similar aggregates were present in the HD-iPS cells during differentiation. We next used immunofluorescence to evaluate the appearance of EM48positive HTT inclusions in HD-iPS cells during neuronal differentiation at days $0,10,15$, and 21 . Although the wt and HD fibroblasts as well as the proliferating wt- and HD-iPS cells did not display immunopositive HTT aggregates, we occasionally detected EM48-positive HTT inclusions in GAP-43 positive mature neurons in three HD-iPS cell clones starting from day 10 of neuronal differentiation (Figs. 6A and $\mathrm{B}$ ). The observation that cultured fibroblasts and pluripotent cells were devoid of aggregates while differentiating cells began to manifest this phenotype implies that a cell-specific process allows retention of the mutant protein in an aggregate-prone state.

\section{Discussion}

The advent of iPS cell technology (Takahashi and Yamanaka, 2006) has opened the door to generating patient-specific sources of donor cells for transplantation approaches that may preclude immunorejection, although a cautionary note to this aspect was recently raised (Zhao et al., 2011). More realistic hopes lie in the unprecedented opportunity to recapitulate pathological tissue formation in vitro, thereby enabling the investigation of complex questions related to neurobiology, disease-specific processes, and drug development. 
A

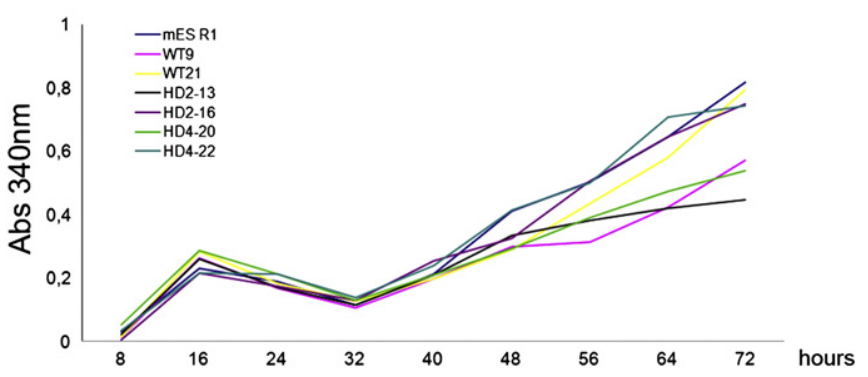

B

wt-iPS

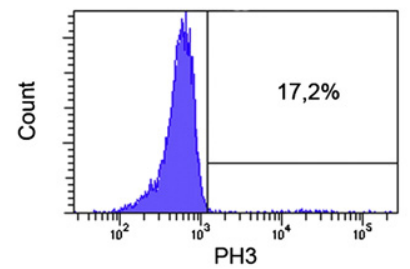

HD-iPS
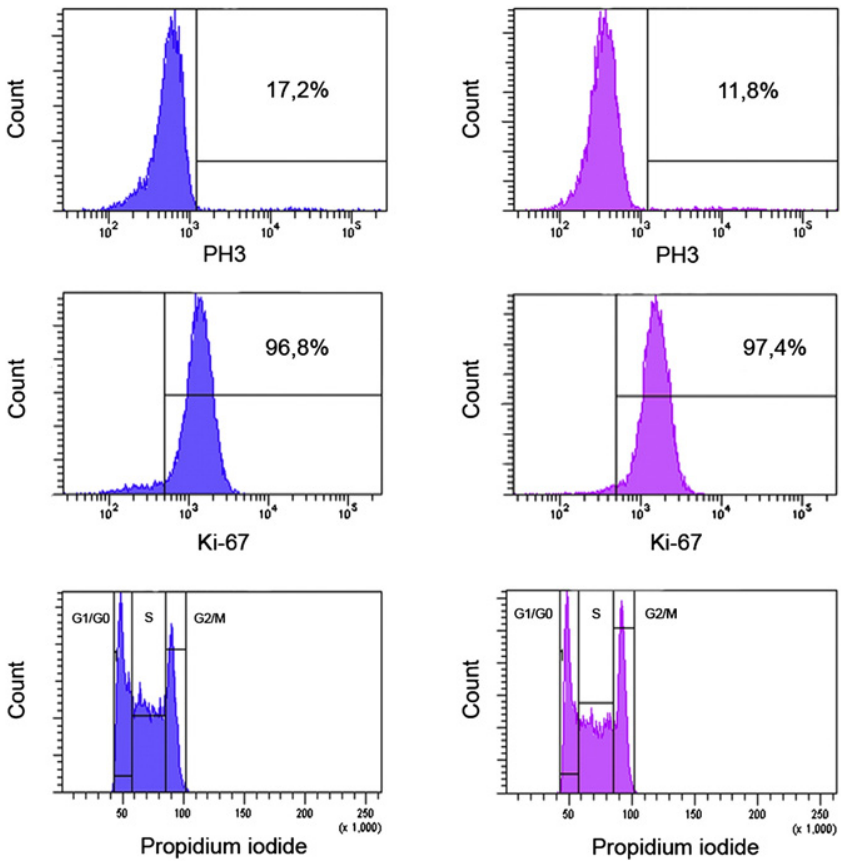

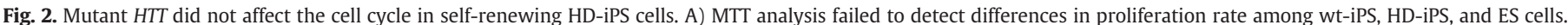

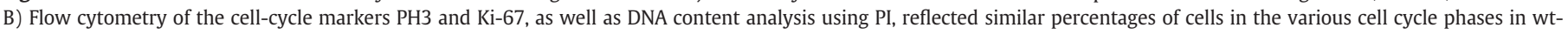
and HD-iPS cells.

Several disease-specific iPS cell lines have been generated from patients suffering various neurological disorders, such as amyotrophic lateral sclerosis (Dimos et al., 2008), spinal muscular atrophy (Ebert et al., 2009), familial dysautonomia (Lee and Studer, 2009), Parkinson's disease, Down syndrome (Park et al., 2008; Seibler et al., 2011; Soldner et al., 2009), Rett syndrome (Marchetto et al., 2010), and schizophrenia (Brennand et al., 2011). One iPS cell line was generated from a single HD patient (Park et al., 2008), but no phenotypes were investigated; more recently, neural stem cells derived from that single HD-iPS line displayed altered levels of caspase activity after serum withdrawal compared to normal neural stem cells (Zhang et al., 2010). HD-iPS cells have also been obtained from monkeys and the presence of HTT aggregates was detected in iPS cell neural derivatives (Chan et al., 2010).

Here we report the first generation and characterization of a large number of iPS cell lines from fibroblasts derived from the R6/2 HD mouse model. The HD- and wt-iPS cells are characterized by similar behaviors in terms of expression profile, proliferation, and differentiation potential. CAG expansion had no detectable effect on the timing and efficiency of the reprogramming process in HD lines compared to the wt lines, leading to a similar number of iPS cell colonies three weeks after viral infection. No differences were observed in iPS cell colony morphology or in the expression of pluripotency markers in all the wt- and HD-iPS cell lines tested, up to 25 passages in vitro. These observations are in agreement with previous studies of human and monkey HD-iPS cell lines that reported no alterations in the reprogramming process or in the maintenance of pluripotency traits (Chan et al., 2010; Zhang et al., 2010).

Our wt- and HD-iPS lines did not differ in terms of their growth rates, cell-cycle progression, or neuronal and glial differentiation. This scenario may not be unexpected for a disease characterized by late onset and slow progression, although a major expectation of iPS cell technology is the possibility to generate disease-specific cellular tool that mirrors the neurodegeneration observed in the brain. A recent investigation of Parkinson's disease iPS cells reported that the PTEN induced putative kinase 1(PINK1) genetic mutation did not affect the ability of mutant iPS cells to differentiate into neuronal progeny (Seibler et al., 2011).

Analyses of the transcription of Bdnf and certain cholesterogenic genes affected in a large number of disease models also resulted in variable outcomes. For example, we did not observe differences between self-renewing wt- and HD-iPS cells, although the $7 \mathrm{dhcr}$ 


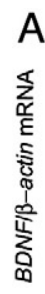
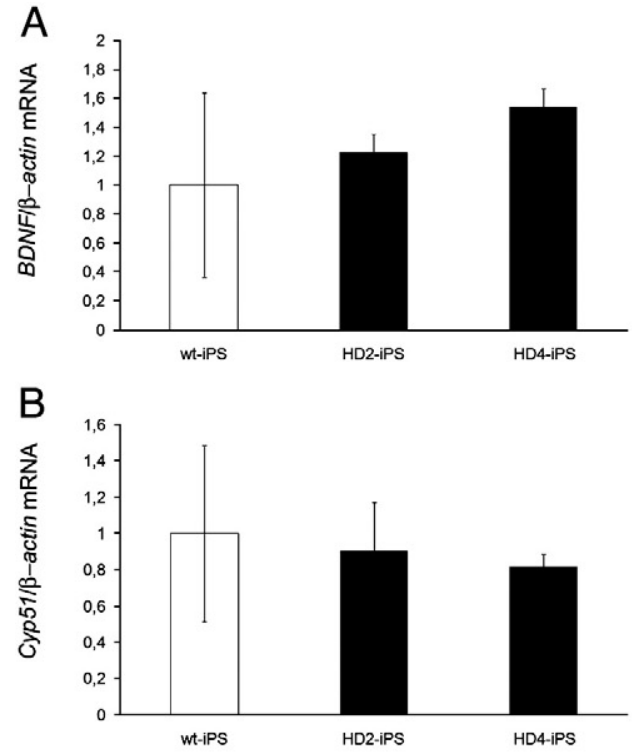

C

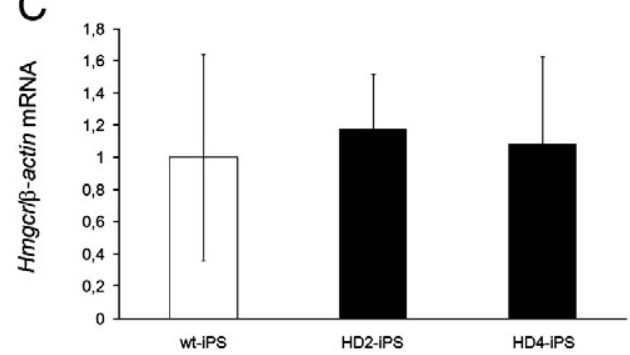

D

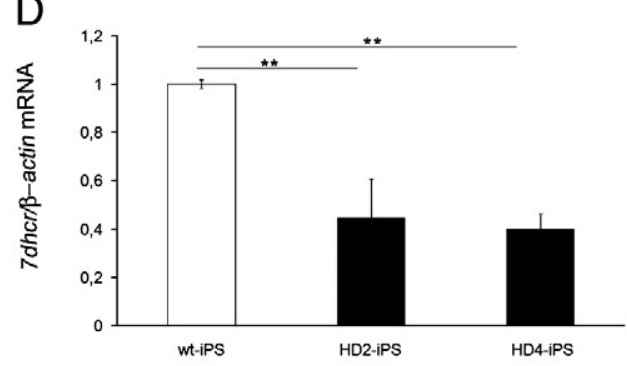

E

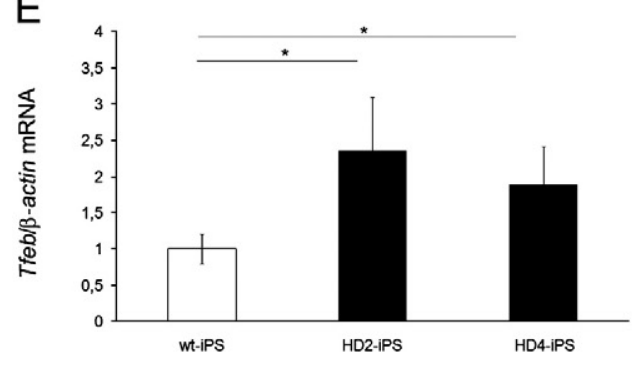

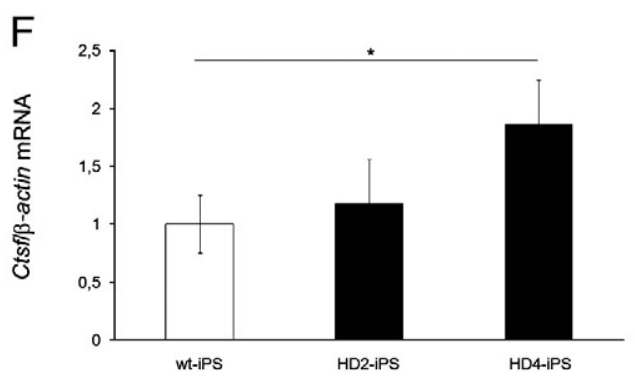

G

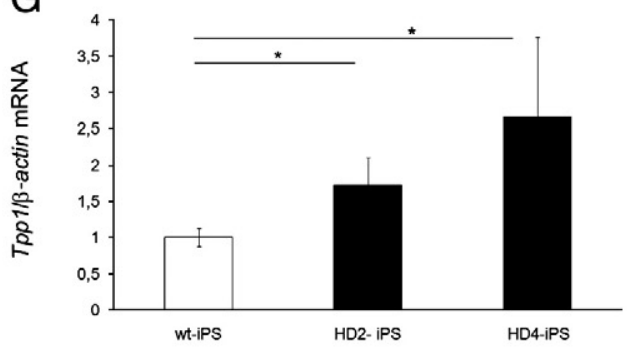

$\mathrm{H}$
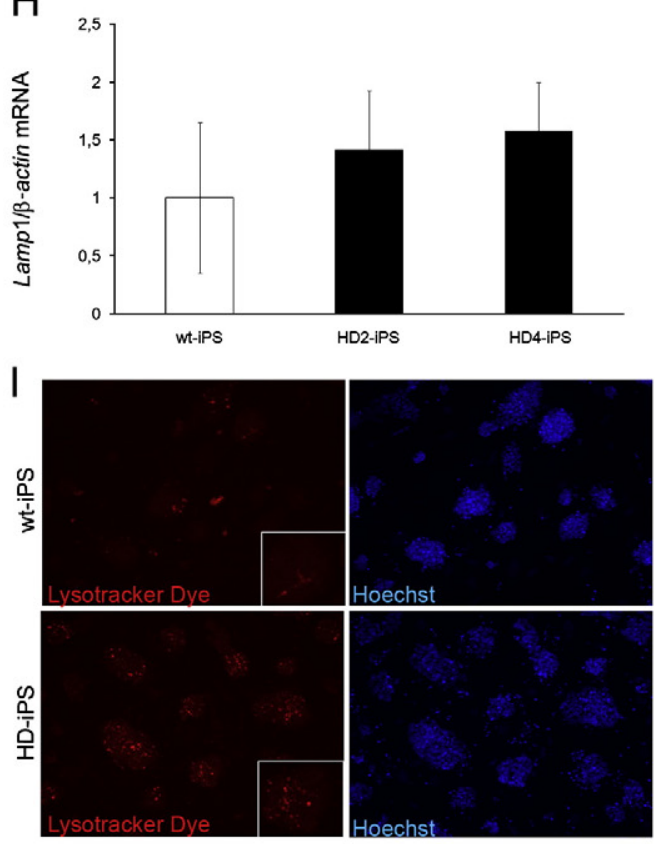

J

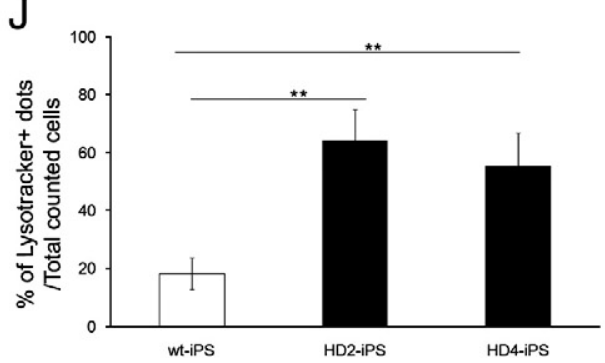

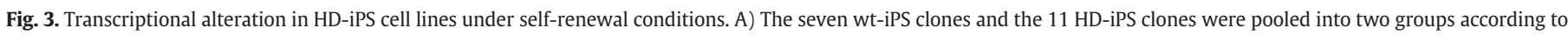

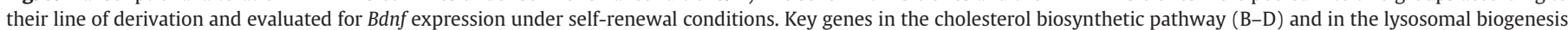

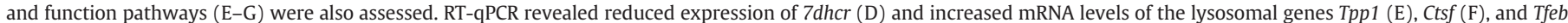

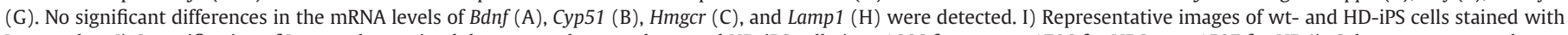

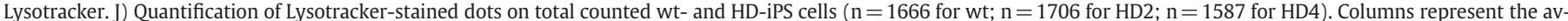

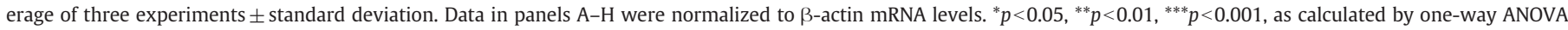
followed by Dunnett post-hoc test.

mRNA level was reduced in HD-iPS cells compared to wt-iPS cells. On the other hand, we detected HTT aggregates in mature neurons, another main feature of $\mathrm{HD}$, starting from day 10 of neuronal differentiation in
HD-iPS cells; no detectable aggregates were observed in undifferentiated HD-iPS cells or primary fibroblasts, a finding that is in agreement with Chan et al. (2010). 


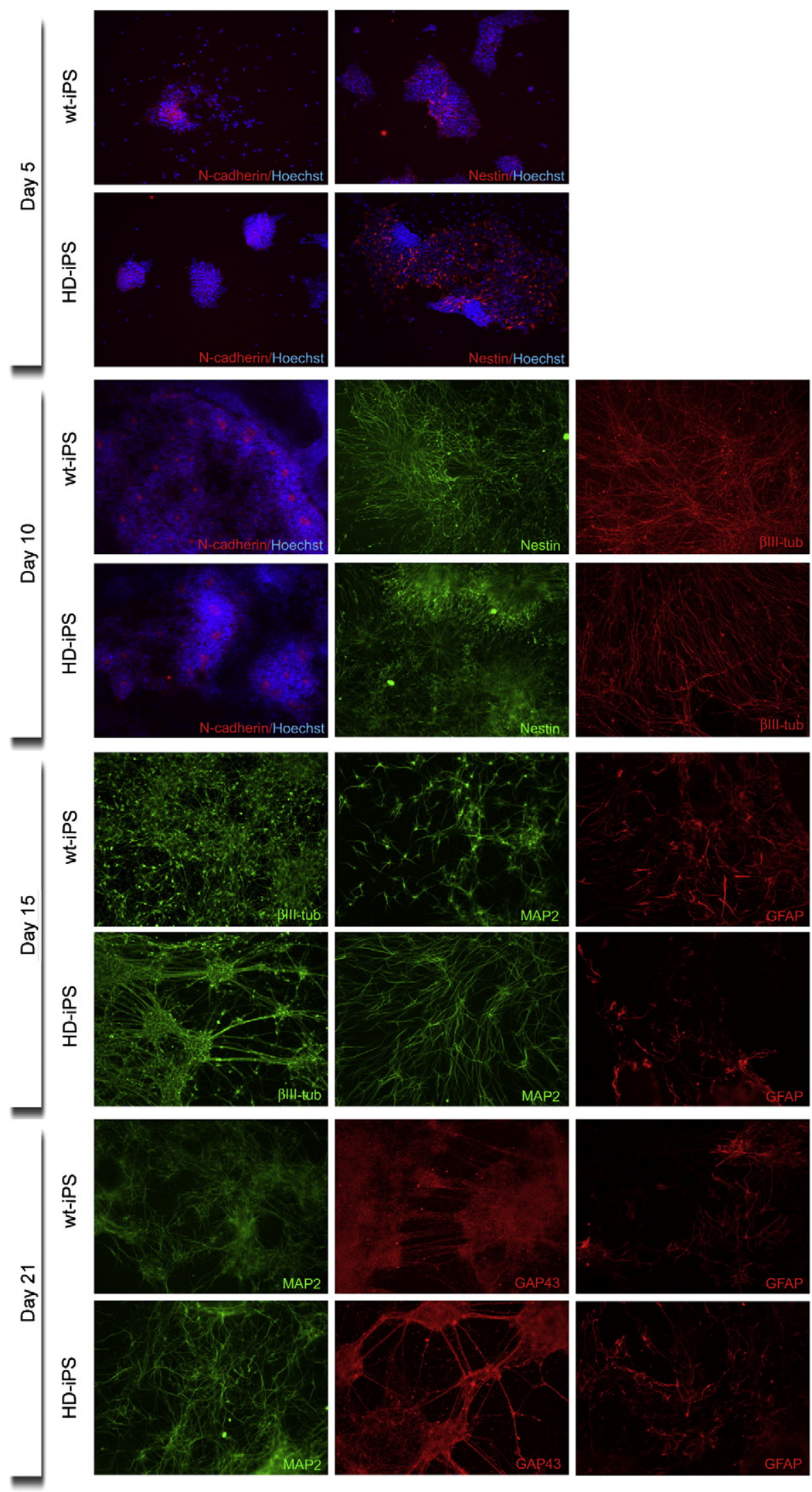

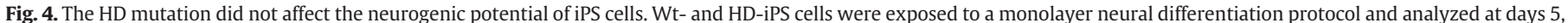

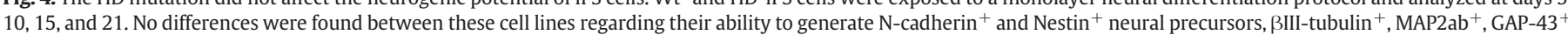
neurons, and $\mathrm{GFAP}^{+}$astrocytes. 


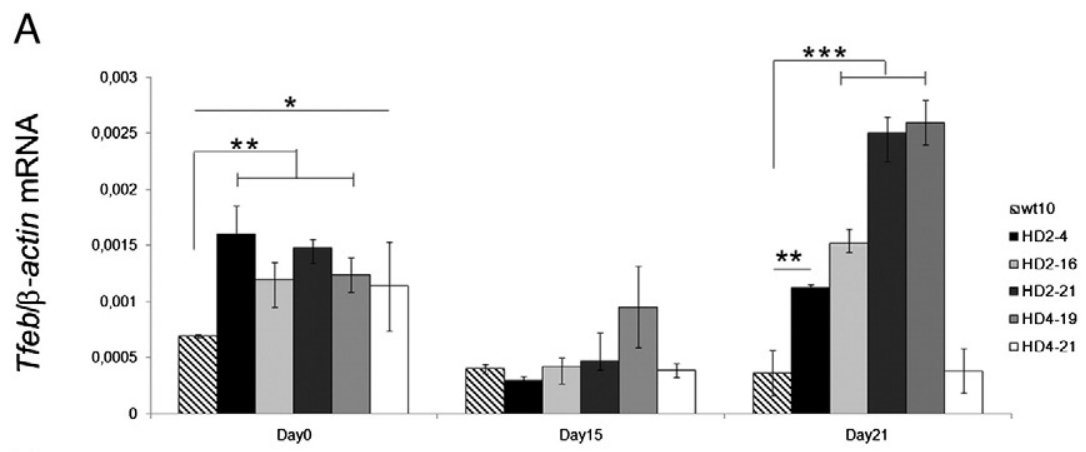

A

B
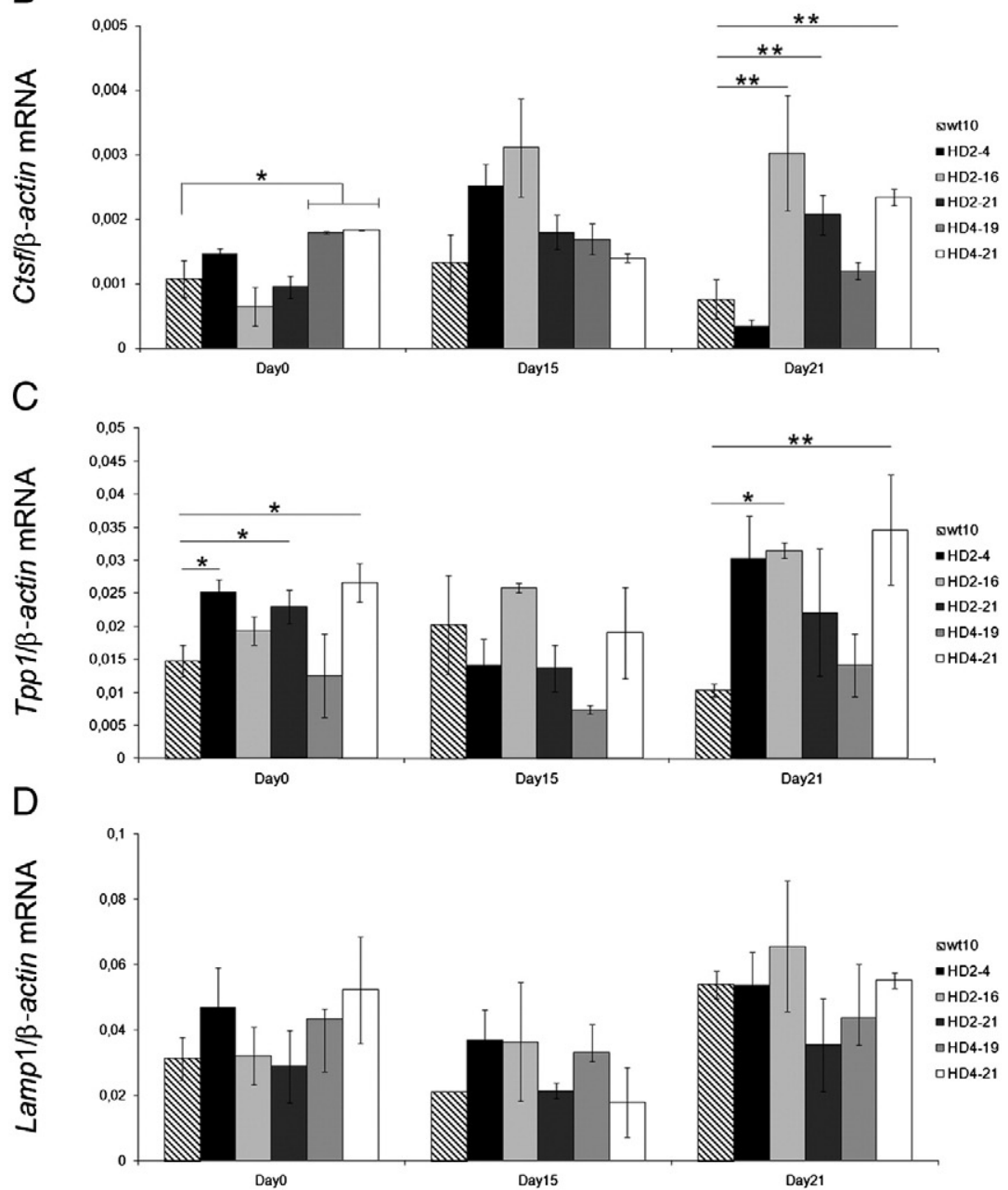

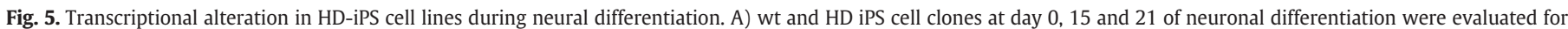

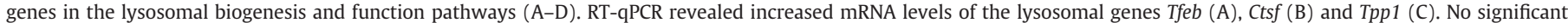

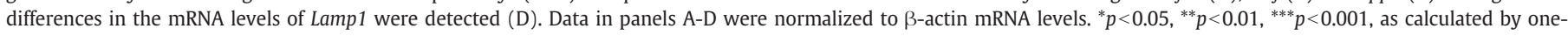
way ANOVA followed by Bonferroni post-hoc test.

Autophagy, a cellular catabolic process that relies on TFEBmediated cooperation between autophagosomes and lysosomes (Sardiello et al., 2009; Settembre et al., 2011), also increases in HD (Ravikumar et al., 2004). We observed upregulation in the mRNA expression of Tfeb and TFEB target genes (Ctsf, Tpp1) in HD-iPS cells versus wt-iPS cells both in self-renewal condition and during neuronal differentiation. This observation suggests that mutant HTT in HD-iPS cells is able to solicit, directly or indirectly, the transcription of Tfeb, the master gene of the autophagic and lysosomal pathways, thus increasing lysosome number. Taken together, these observations suggest that a molecular link between mutant HTT and the transcriptional upregulation of Tfeb and the lysosomal response is present in HD-iPS cells even if additional studies are needed to elucidate the underlying molecular mechanism. Future efforts in the derivation of a collection of human HD-iPS cell lines from various patients could be focused into a platform for the screening of compounds that influence the autophagic and lysosomal processes.

Although the in vitro generation of cell entities reflecting exact human disease phenotypes is not obvious, intriguing cases for iPS cells derived from schizophrenia- (Brennand et al., 2011), familial dysautonomia- (Lee and Studer, 2009), and cardiac syndromes(Moretti et al., 2010) affected patients have been reported. In our experiments, all lines were derived from the same genetic background, possibly increasing the consistency of our data. However, cell 
iPS cell neuronal differentiation

A
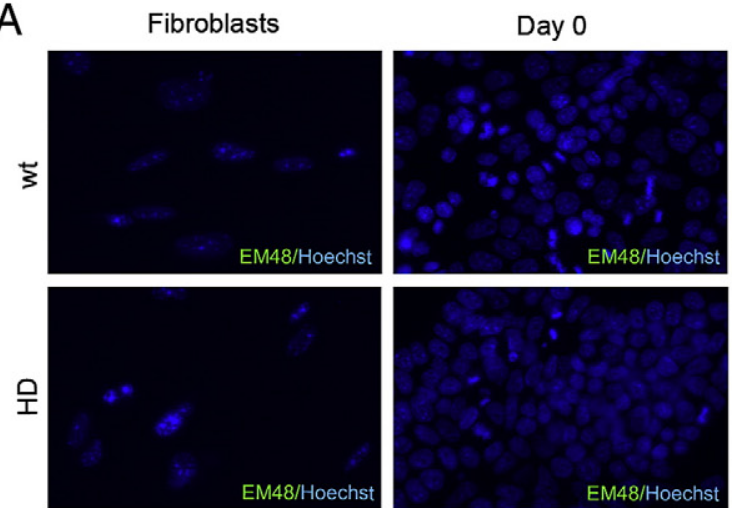

B
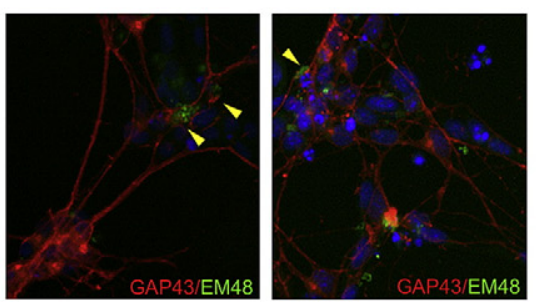

Day 10
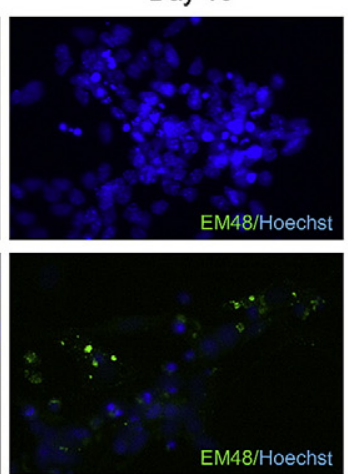

Day 15
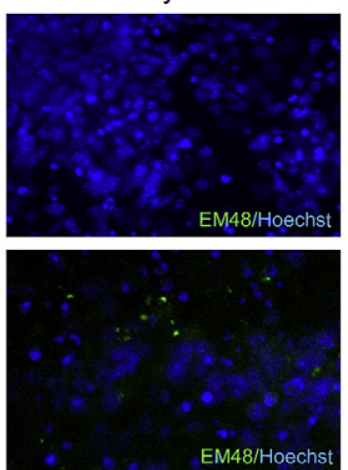

Day 21
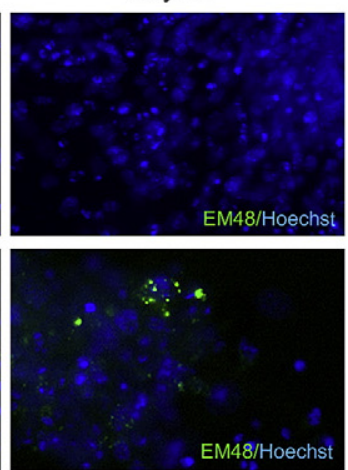

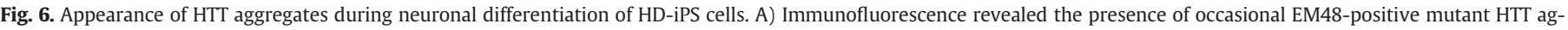

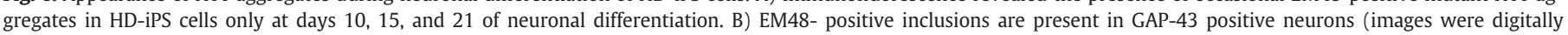
magnified).

variability and the background genetic diversity of the iPS cell lines are aspects that must be considered and implemented when assaying the potential of iPS cell technology for the study of pathogenic mechanisms.

\section{Conclusions}

iPS cells can be generated through somatic reprogramming of readily accessible tissue from patients with any condition. The obvious advantage of such an approach is that patient-specific iPS cells carry the precise genetic variants, both known and unknown, involved in the disease. Additionally, patient-specific iPS cells may eventually serve as a customizable resource for personalized regenerative medicine, drug testing, and predictive toxicology studies. Here we have reported a proof-of-principle application of iPS cell technology by modeling the Corea of Huntington, an untreatable neurological disease. We investigated a variety of aspects using this HD-iPS cell lines, including cell proliferation, pluripotency-marker expression, neurogenic and gliogenic differentiation potential, and the appearance of HTT aggregates in a large number of wt and HD mouse iPS cell lines. While some relevant features of the disease were recapitulated, others were not, suggesting that HD-iPS cells may represent a suitable tool for targeted cellular and molecular investigations of HD mechanisms and screening opportunities, provided that a thorough assessment of the genetic and random variable behavior of multiple lines is fully considered.

Supplementary materials related to this article can be found online at doi:10.1016/j.nbd.2011.12.032.

Supplementary data to this article can be found online at doi:10. 1016/j.nbd.2011.12.032.

\section{Acknowledgments}

This research was supported by initial funding from Fondazione Cariplo (Italy) in the context of the Operational Network for Biomedicine par Excellence in Lombardy project entitled "A genetic toolkit for the analyses of neural stem cells-acronym: Mouse NS-toolkit." This work was also supported by Ministero dell'Istruzione dell'Universita`e della Ricerca (MIUR, 2008JKSHKN to E. Cattaneo) and partially by NeuroStemcell (European Community's Seventh Framework Programme grant agreement nr. 222943). We acknowledge the contributions of Unicredit Banca S.p.A. (Italy) and Tavola Valdese (Italy). We also thank the families of patients with HD for their continuous support.

\section{References}

Abeliovich, A., Doege, C.A., 2009. Reprogramming therapeutics: iPS cell prospects for neurodegenerative disease. Neuron 61, 337-339.

Apostol, B.L., et al., 2008. CEP-1347 reduces mutant huntingtin-associated neurotoxicity and restores BDNF levels in R6/2 mice. Mol. Cell. Neurosci. 39, 8-20.

Blelloch, R., et al., 2007. Generation of induced pluripotent stem cells in the absence of drug selection. Cell Stem Cell 1, 245-247.

Brennand, K.J., et al., 2011. Modelling schizophrenia using human induced pluripotent stem cells. Nature 473, 221-225.

Cattaneo, E., 2001. Assessment of cellular proliferation by calculation of mitotic index and by immunohistochemistry. Methods Mol. Med. 57, 123-131.

Cha, J.H., et al., 1998. Altered brain neurotransmitter receptors in transgenic mice expressing a portion of an abnormal human huntington disease gene. Proc. Natl. Acad. Sci. U. S. A. 95, 6480-6485.

Chan, A.W., et al., 2010. Reprogramming Huntington monkey skin cells into pluripotent stem cells. Cell. Reprogram. 12, 509-517.

Conforti, P., et al., 2008. Blood level of brain-derived neurotrophic factor mRNA is progressively reduced in rodent models of Huntington's disease: restoration by the neuroprotective compound CEP-1347. Mol. Cell. Neurosci. 39, 1-7.

Davies, S.W., et al., 1997. Formation of neuronal intranuclear inclusions underlies the neurological dysfunction in mice transgenic for the HD mutation. Cell 90, 537-548.

Dimos, J.T., et al., 2008. Induced pluripotent stem cells generated from patients with ALS can be differentiated into motor neurons. Science 321, 1218-1221.

Ebert, A.D., et al., 2009. Induced pluripotent stem cells from a spinal muscular atrophy patient. Nature 457, 277-280.

Kim, D.S., et al., 2010. Robust enhancement of neural differentiation from human ES and iPS cells regardless of their innate difference in differentiation propensity. Stem Cell Rev. 6, 270-281.

Lee, G., Studer, L., 2009. Modelling familial dysautonomia in human induced pluripotent stem cells. Philos. Trans. R. Soc. Lond. B Biol. Sci. 366, 2286-2296.

Leoni, V., et al., 2011. Whole body cholesterol metabolism is impaired in Huntington's disease. Neurosci. Lett. 494, 245-249.

Luthi-Carter, R., et al., 2000. Decreased expression of striatal signaling genes in a mouse model of Huntington's disease. Hum. Mol. Genet. 9, 1259-1271. 
Luthi-Carter, R., et al., 2002. Dysregulation of gene expression in the R6/2 model of polyglutamine disease: parallel changes in muscle and brain. Hum. Mol. Genet. 11, 1911-1926.

Luthi-Carter, R., et al., 2003. Complex alteration of NMDA receptors in transgenic Huntington's disease mouse brain: analysis of mRNA and protein expression, plasma membrane association, interacting proteins, and phosphorylation. Neurobiol. Dis. $14,624-636$.

Mangiarini, L., et al., 1996. Exon 1 of the HD gene with an expanded CAG repeat is sufficient to cause a progressive neurological phenotype in transgenic mice. Cell 87, 493-506.

Marchetto, M.C., et al., 2010. A model for neural development and treatment of Rett syndrome using human induced pluripotent stem cells. Cell 143, 527-539.

Moretti, A., et al., 2010. Patient-specific induced pluripotent stem-cell models for longQT syndrome. N. Engl. J. Med. 363, 1397-1409.

Morita, S., et al., 2000. Plat-E: an efficient and stable system for transient packaging of retroviruses. Gene Ther. 7, 1063-1066.

Morton, A., 2000. Progressive formation of inclusions in the striatum and hippocampus of mice transgenic for the human Huntington's disease mutation. J. Neurocytol.

Nakagawa, M., et al., 2008. Generation of induced pluripotent stem cells without Myc from mouse and human fibroblasts. Nat. Biotechnol. 26, 101-106.

Onorati, M., et al., 2010. Neuropotent self-renewing neural stem (NS) cells derived from mouse induced pluripotent stem (iPS) cells. Mol. Cell. Neurosci. 43, 287-295.

Park, I.H., et al., 2008. Disease-specific induced pluripotent stem cells. Cell 134, 877-886.

Ravikumar, B., et al., 2002. Aggregate-prone proteins with polyglutamine and polyalanine expansions are degraded by autophagy. Hum. Mol. Genet. 11, 1107-1117.

Ravikumar, B., et al., 2004. Inhibition of mTOR induces autophagy and reduces toxicity of polyglutamine expansions in fly and mouse models of Huntington disease. Nat. Genet. 36 (6), 585-595.

Reiner, A., et al., 1988. Differential loss of striatal projection neurons in Huntington disease. Proc. Natl. Acad. Sci. U. S. A. 85, 5733-5737.

Rigamonti, D., et al., 2000. Wild-type huntingtin protects from apoptosis upstream of caspase-3. J. Neurosci. 20, 3705-3713.

Rigamonti, D., et al., 2007. Loss of huntingtin function complemented by small molecules acting as repressor element 1 /neuron restrictive silencer element silencer modulators. J. Biol. Chem. 282, 24554-24562.

Rosas, H.D., et al., 2003. Evidence for more widespread cerebral pathology in early HD: an MRI-based morphometric analysis. Neurology 60, 1615-1620.

Rosas, H.D., et al., 2005. Regional cortical thinning in preclinical Huntington disease and its relationship to cognition. Neurology 65, 745-747.

Rosas, H.D., et al., 2008. Cerebral cortex and the clinical expression of Huntington's disease: complexity and heterogeneity. Brain 131, 1057-1068.

Sadri-Vakili, G., et al., 2007. Histones associated with downregulated genes are hypoacetylated in Huntington's disease models. Hum. Mol. Genet. 16, 1293-1306.
Sardiello, M., et al., 2009. A gene network regulating lysosomal biogenesis and function. Science 325, 473-477.

Seibler, P., et al., 2011. Mitochondrial Parkin recruitment is impaired in neurons derived from mutant PINK1 induced pluripotent stem cells. J. Neurosci. 31, 5970-5976.

Settembre, C., et al., 2011. TFEB links autophagy to lysosomal biogenesis. Science 332, 1429-1433.

Sipione, S., et al., 2002. Early transcriptional profiles in huntingtin-inducible striatal cells by microarray analyses. Hum. Mol. Genet. 11, 1953-1965.

Soldner, F., et al., 2009. Parkinson's disease patient-derived induced pluripotent stem cells free of viral reprogramming factors. Cell 136, 964-977.

Takahashi, K., Yamanaka, S., 2006. Induction of pluripotent stem cells from mouse embryonic and adult fibroblast cultures by defined factors. Cell 126, 663-676.

Takahashi, K., et al., 2007. Induction of pluripotent stem cells from adult human fibroblasts by defined factors. Cell 131, 861-872.

Tarditi, A., et al., 2006. Early and transient alteration of adenosine A2A receptor signaling in a mouse model of Huntington disease. Neurobiol. Dis. 23, 44-53.

Trettel, F., et al., 2000. Dominant phenotypes produced by the HD mutation in STHdh(Q111) striatal cells. Hum. Mol. Genet. 9, 2799-2809.

Valenza, M., Cattaneo, E., 2011. Emerging roles for cholesterol in Huntington's disease. TINS 34, 474-486.

Valenza, M., et al., 2007. Progressive dysfunction of the cholesterol biosynthesis pathway in the R6/2 mouse model of Huntington's disease. Neurobiol. Dis. 28, 133-142.

Valenza, M., et al., 2010. Cholesterol defect is marked across multiple rodent models of Huntington's disease and is manifest in astrocytes. J. Neurosci. 30, 10844-10850.

Ying, Q.L., et al., 2003. Conversion of embryonic stem cells into neuroectodermal precursors in adherent monoculture. Nat. Biotechnol. 21, 183-186.

Zhang, N., et al., 2010. Characterization of human Huntington's disease cell model from induced pluripotent stem cells. PLoS Curr. 2, RRN1193.

Zhao, T., et al., 2011. Immunogenicity of induced pluripotent stem cells. Nature 474, 212-216.

Zuccato, C., et al., 2001. Loss of huntingtin-mediated BDNF gene transcription in Huntington's disease. Science 293, 493-498.

Zuccato, C., et al., 2003. Huntingtin interacts with REST/NRSF to modulate the transcription of NRSE-controlled neuronal genes. Nat. Genet. 35, 76-83.

Zuccato, C., et al., 2005. Progressive loss of BDNF in a mouse model of Huntington's disease and rescue by BDNF delivery. Pharmacol. Res. 52, 133-139.

Zuccato, C., et al., 2007. Widespread disruption of repressor element-1 silencing transcription factor/neuron-restrictive silencer factor occupancy at its target genes in Huntington's disease. J. Neurosci. 27, 6972-6983.

Zuccato, C., et al., 2010. Molecular mechanisms and potential therapeutical targets in Huntington's disease. Physiol. Rev. 90, 905-981. 